\title{
T-Cell Traffic Jam in Hodgkin's Lymphoma: Pathogenetic and Therapeutic Implications
}

\author{
Claudio Fozza and Maurizio Longinotti \\ Institute of Hematology, University of Sassari, Viale San Pietro 12, 07100 Sassari, Italy \\ Correspondence should be addressed to Claudio Fozza, cfozza@uniss.it \\ Received 30 June 2010; Accepted 27 September 2010 \\ Academic Editor: Thomas G. Gross
}

Copyright ( $) 2011$ C. Fozza and M. Longinotti. This is an open access article distributed under the Creative Commons Attribution License, which permits unrestricted use, distribution, and reproduction in any medium, provided the original work is properly cited.

\begin{abstract}
In hematologic malignancies, the microenvironment is often characterized by nonneoplastic cells with peculiar phenotypic and functional features. This is particularly true in Hodgkin's lymphoma (HL), in which T lymphocytes surrounding Hodgkin's ReedSternberg cells are essentially polarized towards a memory T-helper type 2 phenotype. In this paper we will first evaluate the main processes modulating T-cell recruitment towards the lymph node microenvironment in HL, especially focusing on the role played by cytokines. We will then consider the most relevant mechanisms of immune escape exerted by neoplastic cells in order to evade antitumor immunity. The potential pathogenetic and prognostic impact of regulatory $\mathrm{T}$ cells in such a context will be also described. We will finally overview some of the strategies of cellular immunotherapy applied in patients with HL.
\end{abstract}

\section{Infiltrating Lymphocytes in Hodgkin's Lymphoma}

In hematologic malignancies, a specific pathogenetic role is often played by nonneoplastic cells within the microenvironment. This is particularly true in Hodgkin's lymphoma (HL), in which T lymphocytes surrounding Hodgkin's ReedSternberg cells (HRSCs) show peculiar phenotypic and functional features. A possible impairment of the T-cell compartment has been investigated in HL since the tuberculin purified protein derivative (PPD) skin test was found to be frequently negative in large series of patients, and therefore a dysregulation involving delayed cellular immune responses was hypothesized [1]. Although infiltrating T lymphocytes are essentially polarized towards a memory T-helper type 2 (Th2) phenotype [2], several studies have shown possible differences among the different subtypes of HL. Among the molecules potentially involved in modulating the pattern of T-cell infiltration, TARC (thymus and activation-regulated chemokine), a chemokine able to attract activated Th2 cells, was found on immunohistochemical staining to be highly expressed in most-classical HL, but not in lymphocytepredominant HL and non-Hodgkin lymphomas (NHL).
This different expression may at least partially justify the discrepancies observed among T-cell infiltrates in distinct HL subtypes [3]. Also, the T-cell transcription factor profile was shown to be consistent with the lymphoid compartment of origin of the different HL subtypes. In particular, in classical and nodular lymphocyte-predominant HL, the expression of GATA-3 and T-bet recalls the pattern typical of normal interfollicular T-cells and normal germinal centers, respectively [4]. Interestingly even the T-cell activation profile was compared in patients with lymphocyte-predominant HL with nodular or diffuse pattern. By evaluating CD134 and CD38 expression, it was shown that in the nodular variant, $\mathrm{T}$ cells express markers of transient/early T-cell activation, while the diffuse pattern is characterized by persistent cellular activation, resembling what was observed in T-cell-rich large B-cell NHL [5]. More recently, another report focused on lymphocyte-rich classical HL, showing that this subtype is characterized by a stronger expression of B-cell transcription factors in neoplastic cells and by a follicular T-cell background with features intermediate between the classical and the nodular lymphocyte-predominant variants [6].

The microarray technology was even able to highlight the prognostic significance of the composition of the T-cell 
infiltrate in a cohort of 267 patients. In fact, low numbers of infiltrating CD8+, CD56+, and CD57+ cells and high numbers of granzyme B + and TIA- $1+$ cells were associated with a significantly worse outcome, as outlined by a higher frequency of leukocytosis, B symptoms, advanced clinical stage, and by lower response rates [7].

In order to shed some light on the mechanisms determining the composition of this microenvironment, the expression and function of T-cell homing molecules were specifically evaluated. Chemokine receptors: CXCR3, CXCR4, and CCR7 and adhesion molecules including CD62 ligand were found to be expressed on most T-cells within HL tissues, while the corresponding ligands were expressed on malignant cells and vascular endothelium. These features resemble the mechanisms of $\mathrm{T}$ cell recruitment observed in normal lymph nodes, thus further highlighting the cross talk among neoplastic and nonneoplastic cells within the HL microenvironment [8].

The issue of the clonality of infiltrating $\mathrm{T}$ lymphocytes was also assessed in two different studies. In the first one, $\mathrm{CD} 8+\mathrm{T}$ cells were shown to be a polyclonal population with limited clonal expansion. In fact, although sequence analysis of the $\mathrm{V}$ regions revealed the presence of $\mathrm{CD} 8+$ expansions in all cases, most of these clonal expansions accounted for less than $10 \%$ of the whole CD8+ T cell population. Moreover, a comparison of the $\mathrm{V}$ region sequences did not provide evidence that CD8+ T-cells were driven towards a common antigen [9]. In the second study, the T cells adherent to HRSC were examined by single-cell analysis for the $\mathrm{T}$ cell receptor (TCR) gamma gene in $5 \mathrm{HL}$ patients. While no evidence of a clonal expansion was obtained in four of five patients with classic HL, clonal TCR gamma sequences were detected in the only patient with lymphocyte-predominant HL, suggesting that even in HL patients, neoplastic cells may sometimes act as antigen-presenting cells [10].

A gene expression profile analysis was performed also on peripheral $\mathrm{CD} 3+\mathrm{T}$ cells isolated from untreated patients with HL, showing that this cell subset seems to be involved in a Th1 immune response even in peripheral blood. However, this cell subpopulation, hypothetically supporting the antitumor immunity, appears to be functionally impaired, as shown by the balance in the expression of genes modulating either the cell cycle or the immune response [11].

\section{Cytokine Production in the Hodgkin's Lymphoma Microenvironment}

The release of cytokines within the lymph node microenvironment has been often advocated as possible mediator in the crosstalk among HL neoplastic cells and infiltrating Tcells. The overall pattern of cytokine gene expression has been shown to be similar in classic $\mathrm{HL}$ and reactive lymph nodes, as regards IL-2, IL-4, IL-5, and interferon (IFN)gamma [12]. On the other side, it is known that HRSC can bind $\mathrm{T}$ lymphocytes in an antigen-independent way, based on LFA-3 (lymphocyte function-associated antigen 3) to CD2 and ICAM-1 (intercellular adhesion molecule 1) to LFA-1 interactions. Moreover, they show unusually strong expression of the costimulatory molecules CD80 and CD86, whose ligand CD28 is expressed on T-cells [13]. HRSC cells are also known to produce interleukin-(IL-) 1a, TNFalpha, IL-6, IL-7, IL-9, IL-10, IL-13, and tumor growth factor-(TGF-) beta [14], the latter being characterized by a prominent role in suppressing T-cell responses [15].

More recently, the expression of potentially relevant chemokines and chemokine receptors was studied in lymph nodes from 24 patients with HL and in 5 control lymph nodes by combining in situ hybridization, immunohistochemistry, and flow cytometry. Among the different cytokines tested, RANTES was expressed almost exclusively by $\mathrm{T}$ cells. Among receptors, CCR 3 and CCR 5 were highly expressed in $\mathrm{T}$ cells - the former in both CD4+ and CD8+ cells, while the latter mainly in CD4+ cells. However, all these chemokines and receptors were not detected on neoplastic cells. This preferential distribution seems to suggest a potential but yet not completely explained involvement of these molecules in the leukocyte recruitment towards the HL lymph node [16]. The relationship between the expression on neoplastic cells of some specific CXC and CC chemokines such as MIG, IP10, and TARC and the infiltration of Th1 and Th2 lymphocytes was evaluated in another study, showing that all these chemokines may influence the Th2 polarization typical of HL [17].

Similarly, several interleukins with the relative receptors have been shown to play a role in the interaction among neoplastic and infiltrating cells. After the detection of the IL-3 receptor (IL-3R) on HRSC, it has been hypothesized that these neoplastic cells could be able to increase the production of IL-3 by preactivated T cells, thus suggesting an involvement of IL-3/IL-3R interactions in the neoplastic proliferation through a paracrine mechanism [18]. Moreover, both HL-derived cell lines and primary HRSC from lymph node tissues of HL patients express the IL-7 receptor. As these cell lines show the constitutive production of IL7 and neutralizing anti-IL-7 antibodies induce a relevant inhibition of their basal proliferation, IL-7 seems to be also involved in autocrine circuitries modulating neoplastic cell growth. Moreover, the IL-7/IL-7R axis appears to act as a cofactor for the expansion of regulatory T-cells (Treg) and as a potential enhancer for the microenvironmental production of IL-6, a cytokine associated with the presence of "B" symptoms and poor outcome in HL [19]. Interestingly, in HL patients, also peripheral $\mathrm{T}$ lymphocytes have been shown to display an atypical profile of cytokine production, characterized by reduced intracellular IL-2, TNF-alpha, and IFN-gamma and increased cytoplasmic IL-4 production, being the $\mathrm{CD} 3+\mathrm{CD} 8+$ subpopulation specifically responsible for the increased levels of IL-4 $[20,21]$.

\section{Mechanisms of Immune Escape by Neoplastic Cells}

Considering that neoplastic cells are able to proliferate in HL although their microenvironment is characterized by an extensive inflammatory infiltrate, the mechanisms of immune escape have been deeply investigated in this 
disorder. For instance, one of the potentially involved molecules is the immunoregulatory glycan-binding protein galectin-1 (Gal1) which has been shown to be selectively overexpressed on HRSC. In fact, blockade of Gall was able to restore the Th1/Th2 balance which is usually inverted in HL, whereas treatment of activated T-cells with Gall favored the secretion of Th2 cytokines and the expansion of CD4+CD25 high forkhead box P3 (FOXP3)+ Treg. This finding therefore implies that Gall is involved in the development and maintenance of an immunosuppressive Th2/Tregskewed microenvironment [22]. The role of this molecule was also examined in the context of Epstein-Barr virus(EBV-) specific CD8+ T-cell responses in HL. Its expression was associated with a reduced CD8+ T-cell infiltration and more specifically with an impaired response towards latent membrane proteins (LMP) 1 and 2. Moreover, the in vitro exposure to recombinant Gal-1 inhibited proliferation and interferon-gamma expression by EBV-specific T cells [23].

Also the possible role played by the PD-1 (programmed death-1) protein was explored. In fact, this molecule and its ligand are thought to be involved in the functional impairment of $\mathrm{T}$ cells in chronic viral infections or tumor immune evasion. HL and T cell NHL, but not B-cell NHL, were shown to overexpress PD-1 ligand, while PD-1 was markedly elevated in tumor-infiltrating and peripheral $\mathrm{T}$ cells of HL patients. Moreover, blockade of the PD-1 system was able to restore the IFN-gamma production by HLinfiltrating T cells [24]. Using a genome-wide transcriptional approach, CD4+ T cells in HL but not in follicular NHL were demonstrated to be under the inhibitory influence of both TGF-beta and PD-1 in vivo [25]. An increase in the number of PD-1+ lymphocytes, measured within a tissue microarray platform, was also shown to be a stageindependent negative prognostic factor of overall survival as opposed to the number of FOXP3+ Treg [26]. All these findings seem to suggest that the impairment of the cellular immunity typical of HL can mirror a T cell exhaustion, which is at least partially mediated by the PD- 1 signaling pathway.

It is worth noting that very recently an increased number of tumor-associated macrophages was shown by geneexpression profiling to be significantly associated with primary treatment failure, shortened progression-free survival, increased likelihood of relapse after autologous hematopoietic stem cell transplantation, and shortened disease-specific survival in a large cohort of HL patients [27].

Several other molecules have been tested for their possible involvement in such a context. For instance, Prostaglandin E2 has been shown to impair CD4+ T-cell activation by interfering with the mechanisms of intracellular transduction downstream TCR and CD28 [28]. Another possible mechanism of T-cell activation impairment was identified in the reduced expression by HRSC of the B7 proteins [29]. Tissue inhibitor of metalloproteinases 1 (TIMP1) is a protein with proteinase-inhibiting and cytokine properties which has been advocated not only as a survival factor for HRSC but, even more importantly, as potential immunosuppressive agent. In fact, in situ hybridization showed TIMP-1 RNA expression on HRSC, while TIMP-1 production by HRSC cells was demonstrated on immunohistochemical analysis. TIMP-1 was also shown to inhibit T cell cytotoxicity both against autologous cells presenting tumorassociated antigens and within allogeneic mixed lymphocyte cultures [30]. Also, the downregulatory molecule cytotoxic T lymphocyte-associated antigen 4 (CTLA-4) was shown to play a possible role, as the proportion of CTLA-4+/CD3+ cells negatively correlated with proliferative activity, IL-2 and IFN-gamma production by T lymphocytes in HL patients [31]. Even CD30, which is typically expressed on HRSC, was shown to inhibit $\mathrm{T}$ cell proliferation. More specifically, antiCD3-stimulated $\mathrm{T}$ cells in the presence of CD30 failed to increase tritium uptake, to express CD25 and CD26 and to produce IL-2. This effect was however, reversed after addition of exogenous IL-2 or pretreatment of HRSC with anti-CD30 [32]. Interestingly, also the intracellular serpin proteinase inhibitor 9 (PI9) was found to be expressed in 10\% of HL patients. This protein is the only known inhibitor of the proteolytic activity of granzyme B, the primary mediator of apoptosis induced by cytotoxic $\mathrm{T}$ lymphocytes and natural killer (NK) cells, thus suggesting another possible mechanism by which neoplastic cells could interfere with antitumor immunity in HL [33]. At least in other subtypes of lymphoma indoleamine 2,3-dioxygenase has been shown to be specifically, involved in the mechanisms of tumor resistance to chemoimmunotherapeutic treatments, likely due to an abnormally increased stimulation of tryptophan catabolism and to the consequent inhibition of antitumor immunity $[34,35]$.

\section{Role of Regulatory T Cells}

Treg represent a small fraction of peripheral CD4+ T cells which plays a crucial role in the maintenance of immune tolerance [36]. More specifically, they have been shown to influence the susceptibility to and the evolution of infective, neoplastic, and autoimmune diseases [37-39]. The possible involvement of $\mathrm{T}$ cells with immunomodulatory properties in HL pathogenesis was firstly hypothesized in 2004. Infiltrating T cells were shown to be anergic to different kinds of stimulation and to suppress peripheral mononuclear cells when cocultured. Furthermore, flow cytometry demonstrated a high frequency of both IL-10 and CD4+CD25+ Treg secretion in the lymph nodes of HL patients. Interestingly, their suppressive function was abrogated by IL-10 neutralization, prevention of cell-to-cell contactade and block of CTLA-4 [40]. One year later, some studies firstly suggested a possible positive prognostic impact for Treg in patients with HL. Expression of granzyme B, TIA-1, and FOXP3 was evaluated by immunohistochemistry in tissue microarrays of 257 patients, showing that low infiltration of Treg in association with high infiltration of cytotoxic $\mathrm{T}$ cells could predict an unfavorable outcome [41]. Similarly, a reduced FoxP3/granzyme $\mathrm{B}+$ ratio was shown to predict poor failure free survival [42]. The absolute number of intratumoral FOXP3 + cells studied by immunohistochemistry on tissue microarrays was of independent prognostic significance for failure-free survival and of borderline significance for overall survival in classical HL [43]. All these findings, based on 
samples taken at diagnosis, were confirmed also in a cohort of patients with refractory or relapsed disease [44]. Only one study had results not in line with the above-mentioned data, showing that a high ratio of Treg over Th2 cells may result in a significantly shortened disease-free survival, therefore implying a possible inhibitory effect performed by Treg on antitumor immune responses [45].

Several mechanisms have been considered as possible mediators of Treg recruitment towards the HL microenvironment. For instance, the inhibition of CCR4 by using a chimeric anti-CCR4 monoclonal antibody was shown to deplete CCR4+ $\mathrm{T}$ cells and to inhibit the migration of CD4+CD25+ $\mathrm{T}$ cells in vitro [46]. Also, the aberrant expression of IL-21, apart from regulating STAT3 signaling and protecting HRSC from apoptosis, was demonstrated to attract Treg via regulation of macrophage-inflammatory protein-3alpha MIP-3alpha in HRSC [47]. The EBV infection was similarly considered as a potential mediator of Treg recruitment. In fact, the expression of the EBV nuclear antigen 1 in HRSC was shown to mediate the upregulation of the chemokine CCL2 0 and consequently the migration of Treg [48]. On the other side, some data seem to suggest that the expression of LAG-3 by tumor-infiltrating lymphocytes with regulatory properties could impair the anti-EBV CD8+ immune reaction in HL patients [49]. In another study, $\mathrm{KM}-\mathrm{H} 2$, which was established as an HRSC line, was demonstrated to promote a bidirectional differentiation of CD4+ naive $\mathrm{T}$ cells toward Foxp3+ T-cells and CD4+ cytotoxic lymphocytes, suggesting that neoplastic cells may act within the HL microenvironment as antigen-presenting cells driving the differentiation of T helper cells [50].

The enrichment of Treg among infiltrating lymphocytes has been even suggested as a possible diagnostic marker, useful in distinguishing classical HL from other entities [51]. Increased frequencies of CD4+CD25+ Treg have also been demonstrated on peripheral blood of patients with HL [52]. More recently, apart from CD4+CD25+FoxP3+ Treg, other subfamilies of cells with immunomodulatory properties, such as CD4+CD26- T cells, have been suggested to potentially exert immunomodulatory functions within the lymph node microenvironment of patients with HL [53].

\section{Strategies of Cellular Immunotherapy}

Over the last few years, several studies have tried to establish if the biological impact that $\mathrm{T}$ lymphocytes have on $\mathrm{HL}$ pathogenesis could be translated into potential immunotherapeutic strategies. The demonstration, even in HL patients, of a graft-versus-lymphoma effect in the context of allogeneic hematopoietic stem cell transplantation and donor lymphocyte infusions [54], represents the ideal platform for such an approach. In order to overcome the apparent impairment of the antitumor immunity typical of HL, different mechanisms have been investigated.

In particular, several laboratory studies have addressed the defective interaction among HRSC and T cells observed in course of HL. For instance, it is well known that HRSC preferentially attract Th2 T cells and Treg, therefore generating an immunosuppressed tumor environment. On the other side, CD8+ T cells lack CCR4 and are nonresponsive to chemokines usually expressed on HRSC. In this regard it was shown that a potential approach to make CD8+ T cells able to efficiently migrate towards HRSC was the induced expression of CCR4. Moreover, the concomitant expression of both CCR4 and of an anti-CD30 chimeric antigen receptor even allowed them to enhance tumor control when infused intravenously in mice engrafted with human HL [55]. Another potentially reversible feature of HRSC is the resistance to $\mathrm{CD} 8+-$ mediated killing via granzyme $\mathrm{B}$, at least partially due to defects in mitochondrial apoptotic pathways and elevated XIAP (X-linked inhibitor of apoptosis protein) expression. In fact, the expression of the proapoptotic factor Smac and the downregulation of XIAP by RNA interference were demonstrated to enhance the apoptotic response of HRSC as well as their susceptibility to CD8+ cytotoxicity [56]. Also, the stimulation of the zeta chain expression has been explored as potential immunotherapeutic approach in an in vitro study, as HL patients express on their peripheral $\mathrm{T}$ and NK cells reduced levels of this key molecule in TCR activation [57].

Another strategy designed to revert the functional anergy of T cells surrounding HRSC was based on a fusion protein represented by an anti-CD30 antibody targeting also IL2, or IL-12. After binding to CD30+ HL cells, anti-CD30IL-2 or anti-CD30-IL-12, antibody-cytokine fusion proteins were able to induce resting $\mathrm{NK}$ cells, but not $\mathrm{T}$ cells, to lyse the lymphoma cells with very high efficiency [58, 59]. Moreover, as TGF-beta secretion is able to inhibit tumor-specific cellular immunity, cytotoxic T lymphocytes have been engineered with a dominant negative TGF-beta receptor in order to resist the antiproliferative effects exerted by this molecule both in vitro and in vivo [60-62].

A very interesting point of view has been also offered by studies focusing on the role of the so-called sidepopulation, constituted by progenitor cells within the neoplastic population with increased resistance to radiation and chemotherapy. It was shown that both HL cell lines and primary HL tumor samples contain a distinct side cell population, showing increased resistance to gemcitabine but also expressing higher levels of tumor-associated antigens, which could be potentially recognized and killed by specific cytotoxic T lymphocytes [63]. It is worth noting that even the pharmacologic effect of histone deacetylase inhibitors in HL may be associated with the induction of an antitumor activity and with a modulation of the cytokine and chemokine secretion within the lymph node microenvironment [64].

Lastly, cytokine-induced killer cells, that is, peripheral blood mononuclear cells expanded in the presence of IFNgamma, IL-2, and a monoclonal antibody against CD3- have been infused in 7 patients with relapsed HL, showing some limited responses in few of them. Interestingly, these cells share functional and phenotypic properties with both $\mathrm{T}$ cells and NK cells and exhibit nonhuman leukocyte antigen (HLA-) restricted killing of tumor cell targets both in vitro and in vivo [65]. 


\section{EBV Antigens as Potential Targets of Cytotoxic T Cells}

Several studies have highlighted the pathogenetic role of the EBV infection in HL pathogenesis, thus implying that immunotherapeutic approaches could potentially rely on cytotoxic T-cells specifically directed towards EBV antigens. Interestingly, EBV expression seems to influence the composition of the infiltrating lymphocyte population, which on the other side may have an impact on clinical presentation and outcome [66].

As it is known that HLA class I molecules present viral peptides for recognition by $\mathrm{CD} 8+\mathrm{T}$ cells and that this $\mathrm{T}$ cell subset plays a pivotal role in the control of EBV infection [67], several studies have focused on the possible impact on HL pathogenesis of the HLA-mediated EBV antigen presentation. Very interestingly, segments within the HLA class I locus have been demonstrated to be specifically associated with the susceptibility to EBV-positive HL, therefore suggesting that the antigenic presentation of EBVderived peptides is somehow involved in the development of HL [68]. In another study by logistic regression, HLA$A^{*} 01$ alleles were shown to be associated with increased and HLA-A*02 alleles with decreased risk of EBV-related HL. These allele-specific associations correspond to nearly 10fold variation in the risk of HL between HLA-A*01 and HLA-A* 02 homozygotes, pointing once again at the critical role of cytotoxic T-cell responses in EBV-related HL [69].

Interestingly, in the only case of spontaneous remission ever reported in HL, a specific impairment of the antiEBV immunity was described at relapse [70]. The cytotoxic response to EBV latent antigens has been specifically characterized in both blood and tumor-infiltrating lymphocytes of HL patients. Actually EBV-specific CD8+ T cells were detected in blood and biopsy samples from both EBVnegative and EBV-positive patients. However, EBV-specific cytotoxic T-lymphocyte precursors were very rare in the blood of HL patients or even undetectable at the tumor site, further suggesting a possible impairment of the immune responses against EBV [71]. The existence of anti-EBV cytotoxic T cell responses in patients with EBV-negative HL was also confirmed in another study addressing the dynamic variance of this cell population during treatment [72].

The first relevant steps in the clinical setting were made in 2002 when EBV-specific cytotoxic T-lymphocytes were ex vivo generated in 13 patients with relapsed HL, by using autologous EBV-transformed B cells as stimulator cells. The infusion of these cytotoxic T-lymphocytes was followed by an increase in EBV-specific immunity, a decrease in virus load, homing and persistence of CD8+ T cells to sites of malignancy, resolution of constitutional symptoms, and some degree of tumor responses [73-75]. Even better results were obtained by exploiting genetically modified APCs in order to augment the expression and immunogenicity of LMP2 and, therefore, the frequency of LMP2specific cytotoxic T lymphocytes [76]. Very interestingly, also allogeneic anti-EBV cytotoxic $T$ cells have been applied in such a context, showing a good safety profile and once again some clinical responses [77]. Local delivery of IL-12 to tumor sites, by using EBV-specific T cells transduced with a retroviral vector expressing the p40 and p35 subunits of this cytokine, was explored as a possible strategy to overcome the inhibitory effects of the HL microenvironment on cytotoxic T lymphocytes [78].

As patients with active disease display a functional impairment of Ag-specific CD8+ $\mathrm{T}$ cells, some studies have focused on an improved targeting of these CD8+ T cells towards viral antigens expressed in HL. One of these strategies was based on the development of an adenoviral antigen presentation system able to reverse the functional $\mathrm{T}$ cell impairment and restore both IFN-gamma production and cytolytic function. With this approach, activated CD8+ $\mathrm{T}$ cells became able to respond to tumor cells and showed phenotypic features consistent with those of effector $\mathrm{T}$ cells [79]. One of the limits of immunotherapeutic approaches targeting EBV antigens is that a subset of malignant cells in the tumor may lack or lose the expression of these potential targets. EBV-specific cytotoxic T lymphocytes expressing a chimeric antigen receptor specific for CD30 have been designed in order to overcome this problem. These cells, generated from healthy donors and HL patients, were able to kill both EBV-positive and -negative cells and, in a xenograft model, produced antitumor effects against EBV-/CD30+ tumors [80].

\section{Future Perspectives}

Although HL is considered as one of the most curable hematologic malignancies, some relevant issues are yet to be resolved. Firstly, the prognosis of patients which poorly respond to or relapse after first-line treatments is still unsatisfactory. Moreover, identifying therapeutic approaches with minimal toxicity still looks essential in a disorder usually occurring in young adults. Studies further addressing the functional features of the $\mathrm{T}$ cell immune system in $\mathrm{HL}$ patients could not only offer a deeper understanding of the biology of this disease but, much more importantly, help to design new targeted immunotherapeutic strategies with increased specificity and therefore improved efficacy and toxicity profile.

\section{Abbreviations}

CTLA-4: Cytotoxic T lymphocyte-associated antigen 4

EBV: $\quad$ Epstein-Barr virus

FOXP3: Forkhead box P3

Gal1: Galectin-1

HL: Hodgkin's lymphoma

HLA: Human leukocyte antigen

HRSC: Hodgkin's Reed-Sternberg cells

ICAM-1: Intercellular adhesion molecule 1

IFN: Interferon

IL: Interleukin

IL-3R: IL-3 receptor

LFA: Lymphocyte function-associated antigen

LMP: Latent membrane protein

NHL: Non-Hodgkin lymphomas

NK: Natural killer 
PD-1: Programmed death-1

PPD: Purified protein derivative

TARC: Thymus and activation-regulated chemokine

TCR: T-cell receptor

TGF: Tumor growth factor

Th2: T-helper type 2

TIMP: Tissue inhibitor of metalloproteinases

Treg: Regulatory T-cells

XIAP: X-linked inhibitor of apoptosis protein.

\section{References}

[1] A. Rottino and G. T. Hoffmann, "Studies of immunological reactions of patients with Hodgkin's disease: the tuberculin reaction," The American Journal of the Medical Sciences, vol. 220, no. 6, pp. 662-664, 1950.

[2] S. Poppema, "The nature of the lymphocytes surrounding Reed-Sternberg cells in nodular lymphocyte predominance and in other types of Hodgkin's disease," American Journal of Pathology, vol. 135, no. 2, pp. 351-357, 1989.

[3] S. C. Peh, L. H. Kim, and S. Poppema, "TARC, a CC chemokine, is frequently expressed in classic Hodgkin's lymphoma but not in NLP Hodgkin's lymphoma, T-cell-rich B-cell lymphoma, and most cases of anaplastic large cell lymphoma," American Journal of Surgical Pathology, vol. 25, no. 7, pp. 925-929, 2001.

[4] Ç. Atayar, A. van den Berg, T. Blokzijl et al., "Hodgkin's lymphoma associated T-cells exhibit a transcription factor profile consistent with distinct lymphoid compartments," Journal of Clinical Pathology, vol. 60, no. 10, pp. 1092-1097, 2007.

[5] P. Lin, L. J. Medeiros, R. B. Wilder, L. V. Abruzzo, J. T. Manning, and D. Jones, "The activation profile of tumourassociated reactive T-cells differs in the nodular and diffuse patterns of lymphocyte predominant Hodgkin's disease," Histopathology, vol. 44, no. 6, pp. 561-569, 2004.

[6] S. H. Nam-Cha, S. Montes-Moreno, M. T. Salcedo, J. Sanjuan, J. F. Garcia, and M. A. Piris, "Lymphocyte-rich classical hodgkin's lymphoma: distinctive tumor and microenvironment markers," Modern Pathology, vol. 22, no. 8, pp. 10061015, 2009.

[7] T. Álvaro-Naranjo, M. Lejeune, M. T. Salvadó-Usach et al., “Tumor-infiltrating cells as a prognostic factor in Hodgkin's lymphoma: a quantitative tissue microarray study in a large retrospective cohort of 267 patients," Leukemia and Lymphoma, vol. 46, no. 11, pp. 1581-1591, 2005.

[8] L. Machado, R. Jarrett, S. Morgan et al., "Expression and function of T cell homing molecules in Hodgkin's lymphoma," Cancer Immunology, Immunotherapy, vol. 58, no. 1, pp. 85-94, 2009.

[9] L. Trümper, W. Jung, H. Daus, G. Mechtersheimer, F. von Bonin, and M. Pfreundschuh, "Assessment of clonality of rosetting $\mathrm{T}$ lymphocytes in Hodgkin's disease by singlecell polymerase chain reaction: detection of clonality in a polyclonal background in a case of lymphocyte predominance Hodgkin's disease," Annals of Hematology, vol. 80, no. 11, pp. 653-661, 2001.

[10] K. Willenbrock, A. Roers, B. Blohbaum, K. Rajewsky, and M.L. Hansmann, "CD8 ${ }^{+} \mathrm{T}$ cells in Hodgkin's disease tumor tissue are a polyclonal population with limited clonal expansion but little evidence of selection by antigen," American Journal of Pathology, vol. 157, no. 1, pp. 171-175, 2000.
[11] A. Franzke, C. Koenecke, R. Geffers et al., "Classical Hodgkin's lymphoma: molecular evidence for specific alterations in circulating T lymphocytes," Tumor Biology, vol. 27, no. 6, pp. 329-333, 2006.

[12] D. Serrano, F. Ghiotto, S. Roncella et al., "The patterns of IL2, IFN- $\gamma$, IL4 and IL5 gene expression in Hodgkin's disease and reactive lymph nodes are similar," Haematologica, vol. 82, no. 5, pp. 542-549, 1997.

[13] S. Poppema and A. van den Berg, "Interaction between host T cells and Reed-Sternberg cells in Hodgkin lymphomas," Seminars in Cancer Biology, vol. 10, no. 5, pp. 345-350, 2000.

[14] H.-J. Gruss, A. Pinto, J. Duyster, S. Poppema, and F. Herrmann, "Hodgkin's disease: a tumor with disturbed immunological pathways," Immunology Today, vol. 18, no. 4, pp. 156163, 1997.

[15] H. Groux, M. Bigler, J. E. de Vries, and M.-G. Roncarolo, "Interleukin-10 induces a long-term antigen-specific anergic state in human CD4+ T cells," Journal of Experimental Medicine, vol. 184, no. 1, pp. 19-29, 1996.

[16] C. Buri, M. Körner, P. Schärli et al., "CC chemokines and the receptors CCR3 and CCR5 are differentially expressed in the nonneoplastic leukocytic infiltrates of Hodgkin disease," Blood, vol. 97, no. 6, pp. 1543-1548, 2001.

[17] K. Ohshima, T. Tutiya, T. Yamaguchi et al., "Infiltration of Th1 and Th2 lymphocytes around Hodgkin and Reed-Sternberg (H\&RS) cells in Hodgkin disease: relation with expression of CXC and CC chemokines on H\&RS cells," International Journal of Cancer, vol. 98, no. 4, pp. 567-572, 2002.

[18] D. Aldinucci, K. Olivo, D. Lorenzon et al., "The role of interleukin-3 in classical Hodgkin's disease," Leukemia and Lymphoma, vol. 46, no. 3, pp. 303-311, 2005.

[19] L. Cattaruzza, A. Gloghini, K. Olivo et al., "Functional coexpression of Interleukin (IL)-7 and its receptor (IL-7R) on Hodgkin and Reed-Sternberg cells: Involvement of IL-7 in tumor cell growth and microenvironmental interactions of Hodgkin's lymphoma," International Journal of Cancer, vol. 125, no. 5, pp. 1092-1101, 2009.

[20] T. Mainou-Fowler, P. R. A. Taylor, S. Miller, A. M. Dickinson, and S. J. Proctor, "Intracellular cytokine profiles by peripheral blood CD3+ T-cells in patients with classical Hodgkin lymphoma," Leukemia and Lymphoma, vol. 44, no. 8, pp. 13251331, 2003.

[21] T. Mainou-Fowler, S. J. Proctor, and P. R. A. Taylor, "Interleukin 4 production by peripheral blood lymphocytes in patients with classical Hodgkin lymphoma," Leukemia Research, vol. 28, no. 2, pp. 159-166, 2004.

[22] P. Juszczynski, J. Ouyang, S. Monti et al., "The AP1-dependent secretion of galectin-1 by Reed-Sternberg cells fosters immune privilege in classical Hodgkin lymphoma," Proceedings of the National Academy of Sciences of the United States of America, vol. 104, no. 32, pp. 13134-13139, 2007.

[23] M. K. Gandhi, G. Moll, C. Smith et al., "Galectin-1 mediated suppression of Epstein-Barr virus-specific T-cell immunity in classic Hodgkin lymphoma," Blood, vol. 110, no. 4, pp. 13261329, 2007.

[24] R. Yamamoto, M. Nishikori, T. Kitawaki et al., "PD-1 PD-1 ligand interaction contributes to immunosuppressive microenvironment of Hodgkin lymphoma," Blood, vol. 111, no. 6, pp. 3220-3224, 2008.

[25] J. M. Chemnitz, D. Eggle, J. Driesen et al., "RNA fingerprints provide direct evidence for the inhibitory role of TGF $\beta$ and PD-1 on CD4+ T cells in Hodgkin lymphoma," Blood, vol. 110, no. 9, pp. 3226-3233, 2007. 
[26] S. Muenst, S. Hoeller, S. Dirnhofer, and A. Tzankov, "Increased programmed death-1+ tumor-infiltrating lymphocytes in classical Hodgkin lymphoma substantiate reduced overall survival," Human Pathology, vol. 40, no. 12, pp. 1715-1722, 2009.

[27] C. Steidl, T. Lee, S. P. Shah et al., "Tumor-associated macrophages and survival in classic Hodgkin's lymphoma," New England Journal of Medicine, vol. 362, no. 10, pp. 875$885,2010$.

[28] J. M. Chemnitz, J. Driesen, S. Classen et al., "Prostaglandin E2 impairs CD4+ $\mathrm{T}$ cell activation by inhibition of lck: implications in Hodgkin's lymphoma," Cancer Research, vol. 66, no. 2, pp. 1114-1122, 2006.

[29] H. Bosshart, "Levels of B7-2 in tumor cells of Hodgkin's disease," Immunology Letters, vol. 92, no. 3, pp. 217-220, 2004.

[30] E. Oelmann, H. Herbst, M. Zühlsdorf et al., "Tissue inhibitor of metalloproteinases 1 is an autocrine and paracrine survival factor, with additional immune-regulatory functions, expressed by Hodgkin/Reed-Stemberg cells," Blood, vol. 99, no. 1, pp. 258-267, 2002.

[31] A. Kosmaczewska, I. Frydecka, D. Boćko, L. Ciszak, and R. Teodorowska, "Correlation of blood lymphocyte CTLA4 (CD152) induction in Hodgkin's disease with proliferative activity, interleukin 2 and interferon-gamma production," British Journal of Haematology, vol. 118, no. 1, pp. 202-209, 2002.

[32] C.-C. Su, H.-H. Chiu, C.-C. Chang, J.-C. Chen, and S.-M. $\mathrm{Hsu}$, "CD30 Is Involved in Inhibition of T-Cell Proliferation by Hodgkin's Reed-Sternberg Cells," Cancer Research, vol. 64, no. 6, pp. 2148-2152, 2004.

[33] B. A. Bladergroen, C. J. L. M. Meijer, R. L. Ten Berge et al., "Expression of the granzyme B inhibitor, protease inhibitor 9, by tumor cells in patients with non-Hodgkin and Hodgkin lymphoma: A novel protective mechanism for tumor cells to circumvent the immune system?" Blood, vol. 99, no. 1, pp. 232-237, 2002.

[34] A. M. Gasparri, E. Jachetti, B. Colombo et al., "Critical role of indoleamine 2,3-dioxygenase in tumor resistance to repeated treatments with targeted IFN $\gamma$," Molecular Cancer Therapeutics, vol. 7, no. 12, pp. 3859-3866, 2008.

[35] T. Yoshikawa, T. Hara, H. Tsurumi et al., "Serum concentration of L-kynurenine predicts the clinical outcome of patients with diffuse large B-cell lymphoma treated with R-CHOP," European Journal of Haematology, vol. 84, no. 4, pp. 304-309, 2010.

[36] M. Itoh, T. Takahashi, N. Sakaguchi et al., "Thymus and autoimmunity: production of $\mathrm{CD} 25^{+} \mathrm{CD} 4^{+}$naturally anergic and suppressive $\mathrm{T}$ cells as a key function of the thymus in maintaining immunologic self-tolerance," Journal of Immunology, vol. 162, no. 9, pp. 5317-5326, 1999.

[37] S. Hori, T. L. Carvalho, and J. Demengeot, "CD $25^{+} \mathrm{CD} 4^{+}$ regulatory $\mathrm{T}$ cells suppress $\mathrm{CD}^{+} \mathrm{T}$ cell-mediated pulmonary hyperinflammation driven by Pneumocystis carinii in immunodeficient mice," European Journal of Immunology, vol. 32, no. 5, pp. 1282-1291, 2002.

[38] F. Ichihara, K. Kono, A. Takahashi, H. Kawaida, H. Sugai, and H. Fujii, "Increased populations of regulatory T cells in peripheral blood and tumor-infiltrating lymphocytes in patients with gastric and esophageal cancers," Clinical Cancer Research, vol. 9, no. 12, pp. 4404-4408, 2003.

[39] M. R. Ehrenstein, J. G. Evans, A. Singh et al., "Compromised function of regulatory $\mathrm{T}$ cells in rheumatoid arthritis and reversal by anti-TNF $\alpha$ therapy," Journal of Experimental Medicine, vol. 200, no. 3, pp. 277-285, 2004.
[40] N. A. Marshall, L. E. Christie, L. R. Munro et al., "Immunosuppressive regulatory $\mathrm{T}$ cells are abundant in the reactive lymphocytes of Hodgkin lymphoma," Blood, vol. 103, no. 5, pp. 1755-1762, 2004.

[41] T. Álvaro, M. Lejeune, MA. T. Salvadó et al., "Outcome in Hodgkin's lymphoma can be predicted from the presence of accompanying cytotoxic and regulatory T cells," Clinical Cancer Research, vol. 11, no. 4, pp. 1467-1473, 2005.

[42] T. W. Kelley, B. Pohlman, P. Elson, and E. D. Hsi, "The ratio of FOXP3 + regulatory $\mathrm{T}$ cells to granzyme $\mathrm{B}+$ cytotoxic $\mathrm{T} / \mathrm{NK}$ cells predicts prognosis in classical Hodgkin lymphoma and is independent of bcl-2 and MAL expression," American Journal of Clinical Pathology, vol. 128, no. 6, pp. 958-965, 2007.

[43] A. Tzankov, C. Meier, P. Hirschmann, P. Went, S. A. Pileri, and S. Dirnhofer, "Correlation of high numbers of intratumoral FOXP3+ regulatory $\mathrm{T}$ cells with improved survival in germinal center-like diffuse large B-cell lymphoma, follicular lymphoma and classical Hodgkin's lymphoma," Haematologica, vol. 93, no. 2, pp. 193-200, 2008.

[44] A. F. Koreishi, A. J. Saenz, D. O. Persky et al., "The role of cytotoxic and regulatory $\mathrm{T}$ cells in relapsed/refractory hodgkin lymphoma," Applied Immunohistochemistry and Molecular Morphology, vol. 18, no. 3, pp. 206-211, 2010.

[45] S. Schreck, D. Friebel, M. Buettner et al., "Prognostic impact of tumour-infiltrating Th2 and regulatory T cells in classical Hodgkin Lymphoma," Hematological Oncology, vol. 27, no. 1, pp. 31-39, 2009.

[46] T. Ishida, T. Ishii, A. Inagaki et al., "Specific recruitment of CC chemokine receptor 4-positive regulatory T cells in Hodgkin lymphoma fosters immune privilege," Cancer Research, vol. 66, no. 11, pp. 5716-5722, 2006.

[47] B. Lamprecht, S. Kreher, I. Anagnostopoulos et al., "Aberrant expression of the Th2 cytokine IL-21 in Hodgkin lymphoma cells regulates STAT3 signaling and attracts Treg cells via regulation of MIP-3alpha," Blood, vol. 112, no. 8, pp. 33393347, 2008.

[48] K. R. N. Baumforth, A. Birgersdotter, G. M. Reynolds et al., "Expression of the Epstein-Barr virus-encoded Epstein-Barr virus nuclear antigen 1 in Hodgkin's lymphoma cells mediates up-regulation of CCL20 and the migration of regulatory $\mathrm{T}$ cells," American Journal of Pathology, vol. 173, no. 1, pp. 195204, 2008.

[49] M. K. Gandhi, E. Lambley, J. Duraiswamy et al., "Expression of LAG-3 by tumor-infiltrating lymphocytes is coincident with the suppression of latent membrane antigen-specific CD8+ Tcell function in Hodgkin lymphoma patients," Blood, vol. 108, no. 7, pp. 2280-2289, 2006.

[50] T. Tanijiri, T. Shimizu, K. Uehira et al., "Hodgkin's ReedSternberg cell line (KM-H2) promotes a bidirectional differentiation of $\mathrm{CD}^{+} \mathrm{CD} 25^{+} \mathrm{Foxp}^{+} \mathrm{T}$ cells and $\mathrm{CD} 4^{+}$cytotoxic $\mathrm{T}$ lymphocytes from $\mathrm{CD} 4^{+}$naive T cells," Journal of Leukocyte Biology, vol. 82, no. 3, pp. 576-584, 2007.

[51] D. S. Bosler, V. K. Douglas-Nikitin, V. N. Harris, and M. D. Smith, "Detection of T-regulatory cells has a potential role in the diagnosis of classical Hodgkin lymphoma," Cytometry Part B, vol. 74, no. 4, pp. 227-235, 2008.

[52] S. Baráth, M. Aleksza, K. Keresztes et al., "Immunoregulatory $\mathrm{T}$ cells in the peripheral blood of patients with Hodgkin's lymphoma," Acta Haematologica, vol. 116, no. 3, pp. 181-185, 2006.

[53] Y. Ma, L. Visser, T. Blokzijl et al., "The CD4+CD26- Tcell population in classical Hodgkin's lymphoma displays a distinctive regulatory T-cell profile," Laboratory Investigation, vol. 88, no. 5, pp. 482-490, 2008. 
[54] K. S. Peggs, A. Hunter, R. Chopra et al., "Clinical evidence of a graft-versus-Hodgkin's-lymphoma effect after reducedintensity allogeneic transplantation," Lancet, vol. 365, no. 9475, pp. 1934-1941, 2005.

[55] A. Di Stasi, B. De Angelis, C. M. Rooney et al., "T lymphocytes coexpressing CCR4 and a chimeric antigen receptor targeting CD30 have improved homing and antitumor activity in a Hodgkin tumor model," Blood, vol. 113, no. 25, pp. 63926402, 2009.

[56] H. Kashkar, J.-M. Seeger, A. Hombach et al., "XIAP targeting sensitizes Hodgkin lymphoma cells for cytolytic T-cell attack," Blood, vol. 108, no. 10, pp. 3434-3440, 2006.

[57] I. Frydecka, D. Bocko, A. Kosmaczewska, L. Ciszak, and R. Morilla, "The effect of peripheral blood lymphocyte stimulation on zeta chain expression and IL-2 production in Hodgkin's disease," British Journal of Cancer, vol. 84, no. 10, pp. 1339-1343, 2001.

[58] C. Heuser, V. Diehl, H. Abken, and A. Hombach, "Anti-CD30IL-12 antibody-cytokine fusion protein that induces IFN- $\gamma$ secretion of T cells and NK cell-mediated lysis of Hodgkin's lymphoma-derived tumor cells," International Journal of Cancer, vol. 106, no. 4, pp. 545-552, 2003.

[59] C. Heuser, S. Guhlke, A. Matthies et al., "Anti-CD30-scFVFc-IL-2 antibody-cytokine fusion protein that induces resting NK cells to highly efficient cytolysis of Hodgkin's lymphoma derived tumour cells," International Journal of Cancer, vol. 110, no. 3, pp. 386-394, 2004.

[60] C. M. Bollard, C. Rössig, M. Julia Calonge et al., "Adapting a transforming growth factor $\beta$-related tumor protection strategy to enhance antitumor immunity," Blood, vol. 99, no. 9, pp. 3179-3187, 2002.

[61] K. Lacuesta, E. Buza, H. Hauser et al., "Assessing the safety of cytotoxic $\mathrm{T}$ lymphocytes transduced with a dominant negative transforming growth factor- $\beta$ receptor," Journal of Immunotherapy, vol. 29, no. 3, pp. 250-260, 2006.

[62] A. E. Foster, G. Dotti, A. Lu et al., "Antitumor activity of EBVspecific T lymphocytes transduced with a dominant negative TGF- $\beta$ receptor," Journal of Immunotherapy, vol. 31 , no. 5 , pp. 500-505, 2008.

[63] J. A. Shafer, C. R. Cruz, A. M. Leen et al., "Antigen-specific cytotoxic $\mathrm{T}$ lymphocytes can target chemoresistant sidepopulation tumor cells in Hodgkin lymphoma," Leukemia and Lymphoma, vol. 51, no. 5, pp. 870-880, 2010.

[64] D. Buglio, G. V. Georgakis, S. Hanabuchi et al., "Vorinostat inhibits STAT6-mediated TH2 cytokine and TARC production and induces cell death in Hodgkin lymphoma cell lines," Blood, vol. 112, no. 4, pp. 1424-1433, 2008.

[65] T. Leemhuis, S. Wells, C. Scheffold, M. Edinger, and R. S. Negrin, "A phase I trial of autologous cytokine-induced killer cells for the treatment of relapsed Hodgkin disease and non-Hodgkin lymphoma," Biology of Blood and Marrow Transplantation, vol. 11, no. 3, pp. 181-187, 2005.

[66] A. Kandil, S. Bazarbashi, and W. A. Mourad, "The correlation of Epstein-Barr virus expression and lymphocyte subsets with the clinical presentation of nodular sclerosing Hodgkin disease," Cancer, vol. 91, no. 11, pp. 1957-1963, 2001.

[67] A. B. Rickinson and D. J. Moss, "Human cytotoxic T lymphocyte responses to Epstein-Barr virus infection," Annual Review of Immunology, vol. 15, pp. 405-431, 1997.

[68] A. Diepstra, M. Niens, E. Vellenga et al., "Association with HLA class I in Epstein-Barr-virus-positive and with HLA class III in Epstein-Barr-virus-negative Hodgkin's lymphoma," Lancet, vol. 365, no. 9478, pp. 2216-2224, 2005.
[69] H. Hjalgrim, K. Rostgaard, P. C. D. Johnson et al., "HLA-A alleles and infectious mononucleosis suggest a critical role for cytotoxic T-cell response in EBV-related Hodgkin lymphoma," Proceedings of the National Academy of Sciences of the United States of America, vol. 107, no. 14, pp. 6400-6405, 2010.

[70] W. M. Wilson, U. Dua, A. P. Grigg, and M. K. Gandhi, "Correlation of T-cell immune response with spontaneous resolution and subsequent relapse of Hodgkin's lymphoma," Leukemia and Lymphoma, vol. 47, no. 5, pp. 871-876, 2006.

[71] A. L. N. Chapman, A. B. Rickinson, W. A. Thomas, R. F. Jarrett, J. Crocker, and S. P. Lee, "Epstein-barr virus-specific cytotoxic T lymphocyte responses in the blood and tumor site of Hodgkin's disease patients: Implications for a T-cell-based therapy," Cancer Research, vol. 61, no. 16, pp. 6219-6226, 2001.

[72] H. Kohrt, A. Johannsen, R. Hoppe et al., "Dynamic CD8 T-cell responses to tumor-associated Epstein-Barr virus antigens in patients with Epstein-Barr virus-negative Hodgkin's disease," Oncology Research, vol. 18, no. 5-6, pp. 287-292, 2009.

[73] C. M. Rooney, C. Bollard, M. H. Huls et al., "Immunotherapy for Hodgkin's disease," Annals of Hematology, vol. 81, no. 2, pp. S39-S42, 2002.

[74] C. M. Bollard, K. C. M. Straathof, M. H. Huls et al., "The generation and characterization of LMP2-specific CTLs for use as adoptive transfer from patients with relapsed EBVpositive Hodgkin disease," Journal of Immunotherapy, vol. 27, no. 4, pp. 317-327, 2004.

[75] C. M. Bollard, L. Aguilar, K. C. Straathof et al., "Cytotoxic $\mathrm{T}$ lymphocyte therapy for epstein-barr virus+ Hodgkin's disease," Journal of Experimental Medicine, vol. 200, no. 12, pp. 1623-1633, 2004.

[76] C. M. Bollard, S. Gottschalk, A. M. Leen et al., "Complete responses of relapsed lymphoma following genetic modification of tumor-antigen presenting cells and T-lymphocyte transfer," Blood, vol. 110, no. 8, pp. 2838-2845, 2007.

[77] K. G. Lucas, D. Salzman, A. Garcia, and Q. Sun, "Adoptive immunotherapy with allogeneic Epstein-Barr virus (EBV)specific cytotoxic T-lymphocytes for recurrent, EBV-positive Hodgkin disease," Cancer, vol. 100, no. 9, pp. 1892-1901, 2004.

[78] H.-J. Wagner, C. M. Bollard, S. Vigouroux et al., "A strategy for treatment of Epstein-Barr virus-positive Hodgkin's disease by targeting interleukin 12 to the tumor environment using tumor antigen-specific T cells," Cancer Gene Therapy, vol. 11, no. 2, pp. 81-91, 2004.

[79] C. Smith, L. Cooper, M. Burgess et al., "Functional reversion of antigen-specific CD8+ T cells from patients with Hodgkin lymphoma following in vitro stimulation with recombinant polyepitope," Journal of Immunology, vol. 177, no. 7, pp. 48974906, 2006.

[80] B. Savoldo, C. M. Rooney, A. Di Stasi et al., "Epstein Barr virus-specific cytotoxic $\mathrm{T}$ lymphocytes expressing the antiCD30 $\zeta$ artificial chimeric T-cell receptor for immunotherapy of Hodgkin disease," Blood, vol. 110, no. 7, pp. 2620-2630, 2007. 


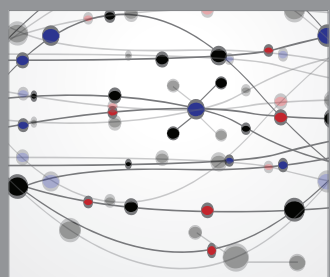

The Scientific World Journal
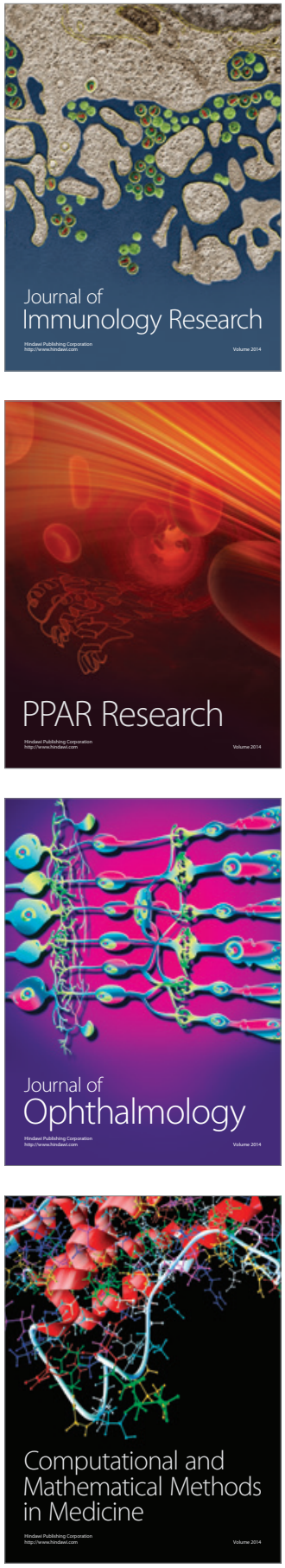

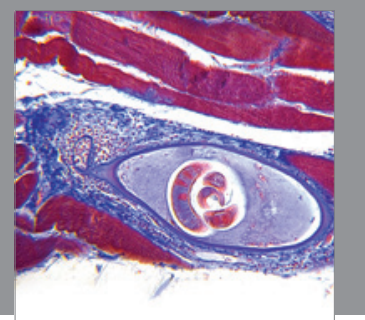

Gastroenterology

Research and Practice
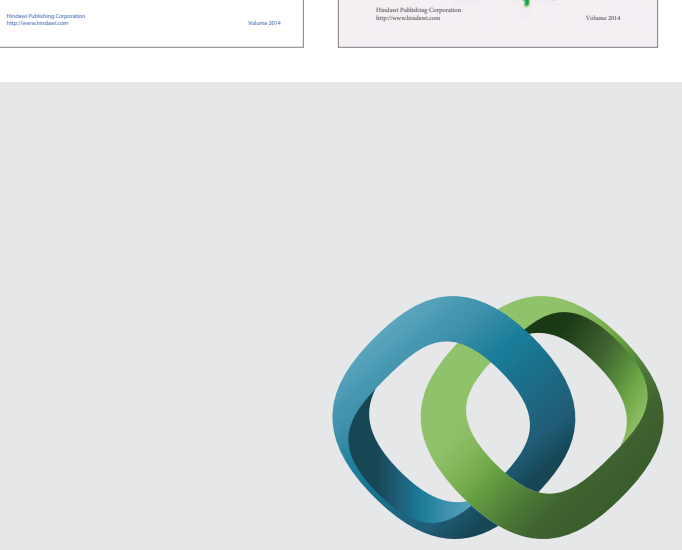

\section{Hindawi}

Submit your manuscripts at

http://www.hindawi.com
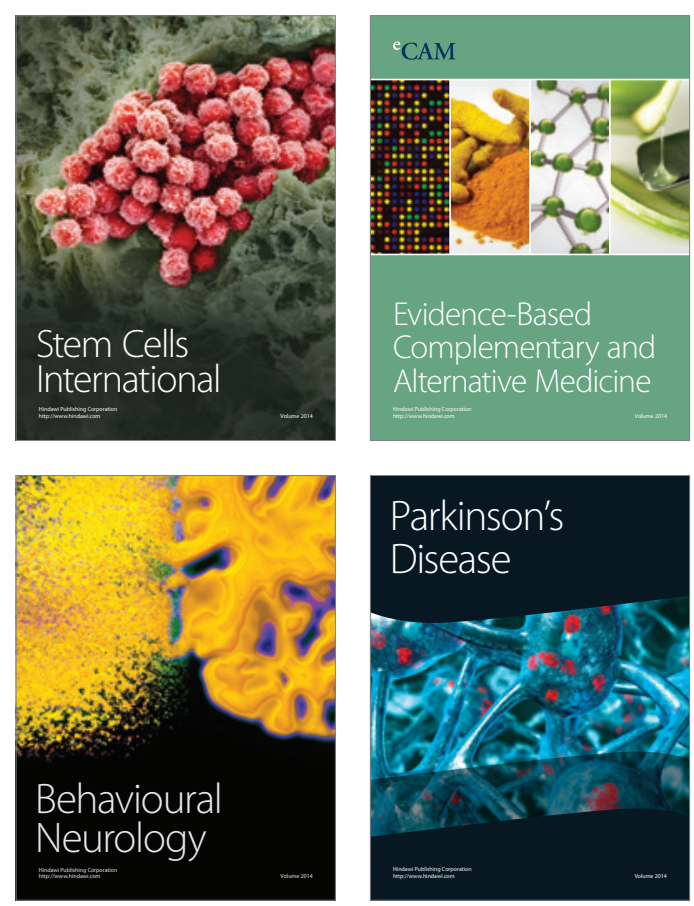

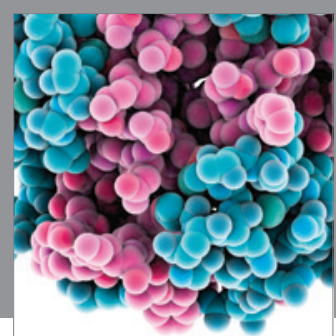

Journal of
Diabetes Research

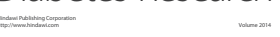

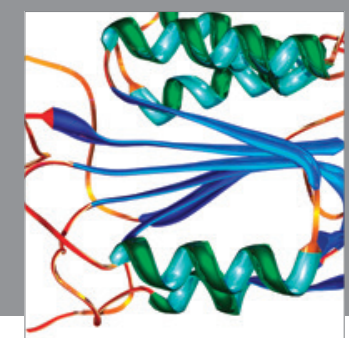

Disease Markers
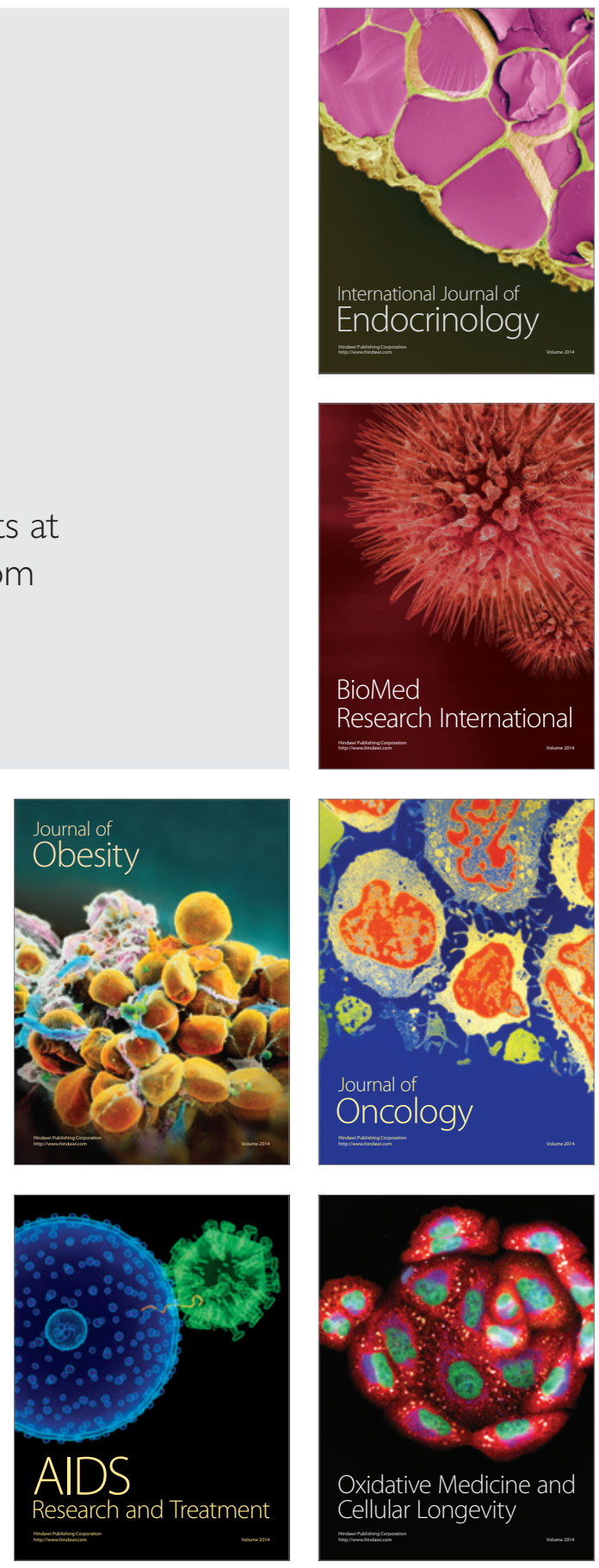